\title{
Feature Extraction for Digging Operation of Excavator Based on Data-Driven Skill-Based PID Controller
}

\author{
Kazushige Koiwai, Toru Yamamoto \\ Institute of Engineering, Hiroshima University, \\ 1-4-1, Kagamiyama, Higashihiroshima City, Hiroshima, 739-8527, Japan \\ E-mail:koiwaik@hiroshima-u.ac.jp,yama@hiroshima-u.ac.jp \\ http://www.hiroshima-u.ac.jp \\ Takao Nanjo, Yoichiro Yamazaki \\ Global Engineering Center, KOBELCO Construction Machinery CO., LTD., \\ 2-2-1, Itsukaichikou, Saeki-ku, Hiroshima City, Hiroshima, 731-5161, Japan
}

\begin{abstract}
Improvement of the work efficiency is demanded by aging and reducing of the working population in the construction field, so that some automation technologies are applied to construction equipment, such as bulldozers and excavators. However, not only the automation technologies but also expert skills are necessary to improve the work efficiency. In this paper, the human skill evaluation is proposed by the data-driven skill-based PID controller. The proposed method is applied to the excavator digging operation. As the result, the difference between the novice operation and the skilled operation is extracted. Moreover, the numerical difference is clarified based on the result.
\end{abstract}

Keywords: PID Controller, Human Skill Evaluation, Data-Driven.

\section{Introduction}

Japanese Ministry of Land, Infrastructure, Transport and Tourism have started the policy of the integration of construction and ICT (Information and Communication Technology) for public works such as the land survey, engineering, the construction and the maintenance, that is called "i-construction". ${ }^{2}$ Aims of this policy are the improvement of the work efficiency and the increasing of productivity. Technologies of IoT (Internet of Things), Big Data and AI (Artificial Intelligence) will be applied to some construction equipments based on the policy. However, human operations for construction equipments are still required continuously in the field because skilled workers optimize their operations for high productivity in each complicated conditions. Unfortunately, those skilled operators who have the unique technique are reduced in these days because of the aging and reducing of the working population.

As the previous studies, skill based controllers have been proposed. ${ }^{2,3}$ These studies considered that the human skill described as a controller with the tuner of
PID gains, called the skill-based PID controller. The operation skill has been replicated by the skill-based PID controller experimentally. Moreover, the skill evaluation by using the skill-based PID controller have been performed. ${ }^{4,5}$ These research focus on the change of PID gains to extract the feature of the operation skill for the excavator. However, the evaluated operation is applied to the simple lever operation such as the swing operation. In the actual work field, the operation for the excavator is more complicated. In the previous study, CMAC is utilized as the parameter tuner. However, CMAC doesn't have extensibility such as the data-driven method.

In this study, the data-driven skill-based PID controller is adopted to the actual work operation of the excavator, i.e. the continuous digging operation. The skill feature for the excavator of the continuous digging operation is extracted to compare the novice operation with the professional operation based on the control engineering approach. Moreover, the numerical difference of feature between is verified. 


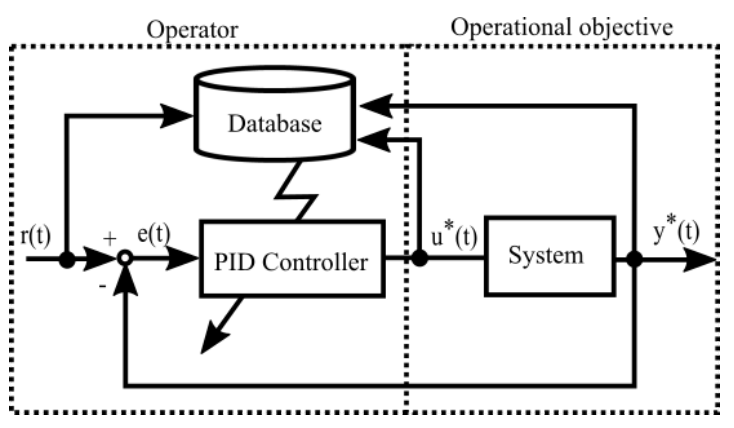

Fig. 1 Scheme of Feature Extraction

\section{Scheme of skill feature extraction}

The scheme of feature extraction for the human skill is shown in Fig. 1. Based on the engineering approach, the operational objective and the operator are considered as the system and the PID controller with the parameter tuner that is consist of the data driven method. $y^{*}(t)$ and $u^{*}(t)$ are denoted as the system output and the system input by the operation.

\subsection{Data-driven PID controller}

The following control law is considered for the skill-based controller:

$\Delta u(t)=K_{I}(t) e(t)-K_{P}(t) \Delta y^{*}(t)-K_{D}(t) \Delta^{2} y^{*}(t)$

where the PID gains, $K_{P}(t), K_{I}(t)$ and $K_{D}(t)$ are tuned by the database. The control error signal $e(t)$ is defined as $e(t):=r(t)-y(t)$ and the difference operator $\Delta$ is defined as $\Delta:=1-z^{-1} . y(t)$ and $u(t)$ are donated as the system output and the system input respectively.

The operational objective is considered as the following nonlinear system:

$$
y^{*}(t)=h(\phi(t-1)) .
$$

Here the $h(\cdot)$ is the nonlinear function using past data and the information vector $\phi(t-1)$ is defined as:

$$
\begin{gathered}
\phi(t-1)=\left[y^{*}(t-1), \cdots y^{*}\left(t-n_{y}\right),\right. \\
\left.u^{*}(t-1), \cdots u^{*}\left(t-n_{u}\right),\right]
\end{gathered}
$$

where $n_{y}$ and $n_{u}$ are orders of output and input, respectively. The query $\bar{\phi}(t)$ is defined as:

$$
\begin{aligned}
\bar{\phi}(t)= & {\left[r(t+1), r(t), y^{*}(t), y^{*}(t-1), \cdots\right.} \\
& \left.y^{*}\left(t-n_{y}\right), u^{*}(t-1), \cdots u^{*}\left(t-n_{u}\right)\right] .
\end{aligned}
$$

The dataset of database, $\boldsymbol{\Phi}(i)$, is constructed as follows:

$$
\begin{gathered}
\boldsymbol{\Phi}(i)=\left[\bar{\phi}(i), K_{P}(i), K_{P}(i), K_{P}(i)\right], \\
i=1,2, \cdots, N
\end{gathered}
$$

where the index and the total number of datasets is shown as $i$ and $\mathrm{N}$, respectively.

PID gains that is requested by the query are calculated by the following procedure. Distances $d(\cdot)$ between the query $\bar{\phi}(t)$ and $\bar{\phi}(i)$ in each datasets are calculated by:

$$
d(\bar{\phi}(t), \bar{\phi}(i))=\sum_{l=1}^{n_{u}+n_{y}+1}\left|\frac{\overline{\phi_{l}}(t)-\overline{\phi_{l}}(i)}{\max \overline{\phi_{l}}-\min \overline{\phi_{l}}}\right|
$$

where the index of an element in a dataset or query is shown as $l . \quad \overline{\boldsymbol{\phi}_{l}}$ is a set of the element for the index.

Neighbors of data are selected according to their distance from query. The number of neighbor dataset, that is the user specified parameter, is donated as $k$. The sum of selected PID gains $\boldsymbol{K}_{\boldsymbol{P I D}}(m)$ multiplied by a weight is desired PID gains $\boldsymbol{K}_{\boldsymbol{P I D}}(t)$ as follows:

$$
\begin{aligned}
& \boldsymbol{K}_{\boldsymbol{P I D}}(t)=\sum_{m=1}^{k} \omega_{m} \boldsymbol{K}_{\boldsymbol{P I D}}(m) \\
& \boldsymbol{K}_{P I D}(t)=\left[\begin{array}{lll}
K_{P}(t) & K_{I}(t) & K_{D}(t)
\end{array}\right] \\
& \omega_{m}=\frac{\left(1+d_{m}\right)^{-1}}{\sum_{n=1}^{k}\left(1+d_{n}\right)^{-1}}
\end{aligned}
$$

\subsection{Skill feature extraction}

PID gains are learned by the operational data $u^{*}(t)$ and $y^{*}(t)$, which are teacher's signals for the learning scheme in Fig. 2. PID controller, which has the change of PID gains can be expressed as the feature of the human skill.

The following steepest descent method (10) is performed for the learning of PID gains in the database.

$$
\boldsymbol{K}_{P I D}^{n e w}(t)=\boldsymbol{K}_{P I D}^{o l d}(t)-g(t) \frac{\partial J(t)}{\partial \boldsymbol{K}_{P I D}}
$$

where the error criterion $J(t)$, the updating gradient $g(t)$ and the partial differential are expressed as follows:

$$
\begin{gathered}
g(t)=\left(c+a \cdot \exp \left(-b\left|u^{*}(t)-u(t)\right|\right)\right)^{-1} \\
J(t)=\frac{\epsilon(t)^{2}}{2} \\
\epsilon(t)=u^{*}(t)-u(t) \\
\frac{\partial J(t)}{\partial \boldsymbol{K}_{P I D}}=\frac{\partial J(t)}{\partial \epsilon(t)} \frac{\partial \epsilon(t)}{\partial u(t)} \frac{\partial u(t)}{\partial \boldsymbol{K}_{P I D}}
\end{gathered}
$$




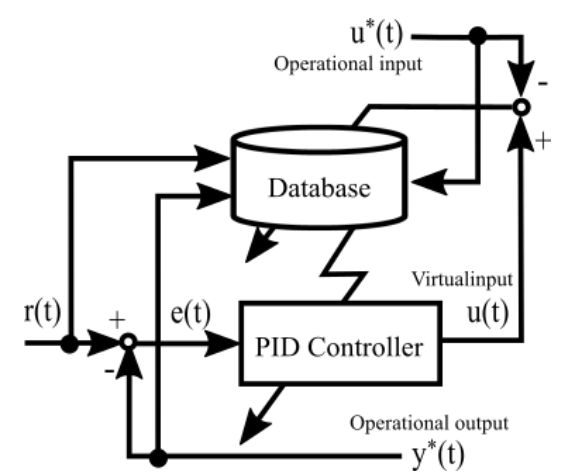

Fig. 2 The Leaning Scheme of Skill Feature Extraction

Coefficients of $a, b$ and $c$ are the appropriate positive constants.

\section{Evaluation Result}

\subsection{Experimental equipment}

The proposed scheme is applied to the operation of an excavator. The evaluated operation is a continuous digging motion and the excavator, SK200-9 made in Kobelco Construction Machinery, is used for the experiment. The continuous digging motion can be classified into four parts in Fig. 3. First part is called digging that is the operation to scoop the earth and sand with the bucket (1). Then, the load is lifted to the certain height that assumed a carrier of the truck (2). Furthermore, the load is dumped on the ground that assumed the truck bed (3). Finally, teeth of the bucket is repositioned to the starting point of the digging (4).

\subsection{Skill evaluation procedure}

The most important operation for actual works is the positioning at the stop motion because the human skill is required by the operation. Therefore, the target for this evaluation is the positioning by the stop operation

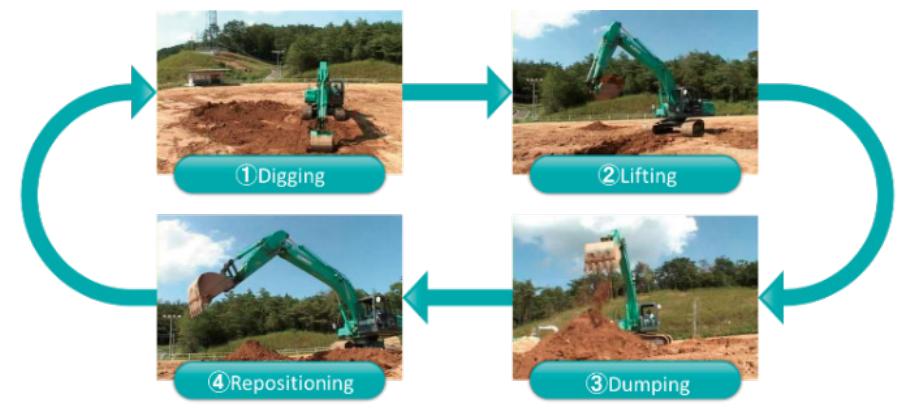

Fig. 3 Continuous Digging Motion for Excavator only. The acceleration should be ignored in this paper. The evaluation procedure is shown as follows.

The proposed method is applied to the specified operation into the continuous digging operation. In this study, the swing operation is selected as the specified operation because the skill is necessary to stop at the proper position and the reference value $r(t)$ of operation is clearer than another operation. The digging operation is performed to record $u^{*}(t)$ and $y^{*}(t)$. Here, the pilot pressure and the angle of the swing are set as the input value $u^{*}(t)$ and the output value $y^{*}(t)$, respectively. Then, the skill feature is extracted by the comparison of the professional and the novice operation. Moreover, the physical quantity that can be measured is evaluated based on the skill feature.

\subsection{Application result of proposed method}

The data of the swing motion in the continuous digging operation is extracted, normalized and resampled by the sampling time of $200 \mathrm{~ms}$ according to the human quickness of a response. The proposed method is applied to the professional and the novice data. The user specific parameter of the proposed method is shown as Table 1.

The calculation results of PID parameter for the professional operator and the novice operator are shown in Fig. 4 and Fig. 5. As the result, the change of PID gain at the period of deceleration which needs the operation skill has the opposite properties to compare with them. This is the almost same result of the skill evaluation for the single operation. Refer to the reference 4 in the detail of the single operation. Especially, derivative gains have the opposite tendency. It means that the professional predicts the future motion based on the property of the derivative gain. Therefore, the professional operation is smoother than the novice operation.

Table 1. User Specified Parameter of database

\begin{tabular}{|l|c|l|}
\hline Item & Symbol & Value \\
\hline Number of data $y$ & $n_{y}$ & 3 \\
\hline Number of data $u$ & $n_{u}$ & 3 \\
\hline Number of neighbor & $k$ & 3 \\
\hline Total number of data set & $\mathrm{N}$ & 100 \\
\hline Coefficient of updating gradient & $a$ & 100 \\
\hline Coefficient of updating gradient & $b$ & 1 \\
\hline Coefficient of updating gradient & $c$ & 100 \\
\hline
\end{tabular}



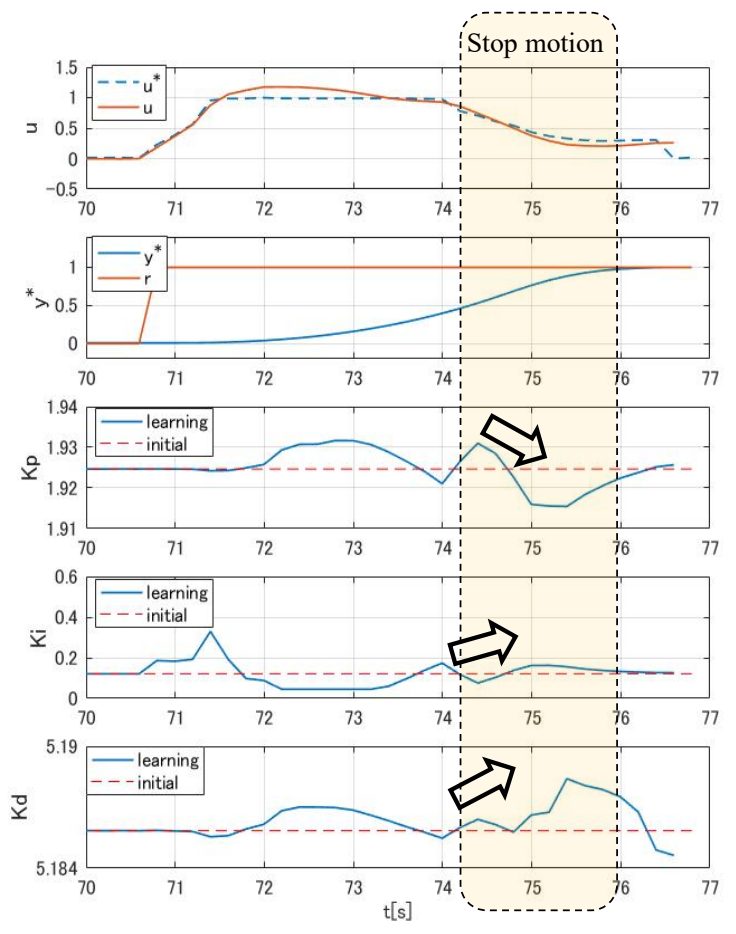

Fig. 4 Professional Skill Feature Extraction

\subsection{Skill numerical evaluation}

The operation of the novice operator is different from the professional one in fluidity because the professional operation is smooth as the above explanation. To compare fluidities, change of input are considered and extracted as the maximum value and the number beyond the $50 \%$ from the 10 cycle of the continuous digging operation. The result is shown as Table 2. As the result, it is verified that the novice has the unnecessary input compared with the professional. It means that the novice operator operates the excavator not so smoothly.

\section{Conclusion}

The human skill evaluation based on the data-driven PID controller has been performed to the excavator operation of the continuous digging motion. The difference between the novice and professional was

Table 2. Comparison of input change

\begin{tabular}{|l|c|c|}
\hline & Professional & Novice \\
\hline Maximum of input change & 0.54 & 0.69 \\
\hline $\begin{array}{l}\text { Number beyond 50\% } \\
\text { input change }\end{array}$ & 1 & 4 \\
\hline
\end{tabular}

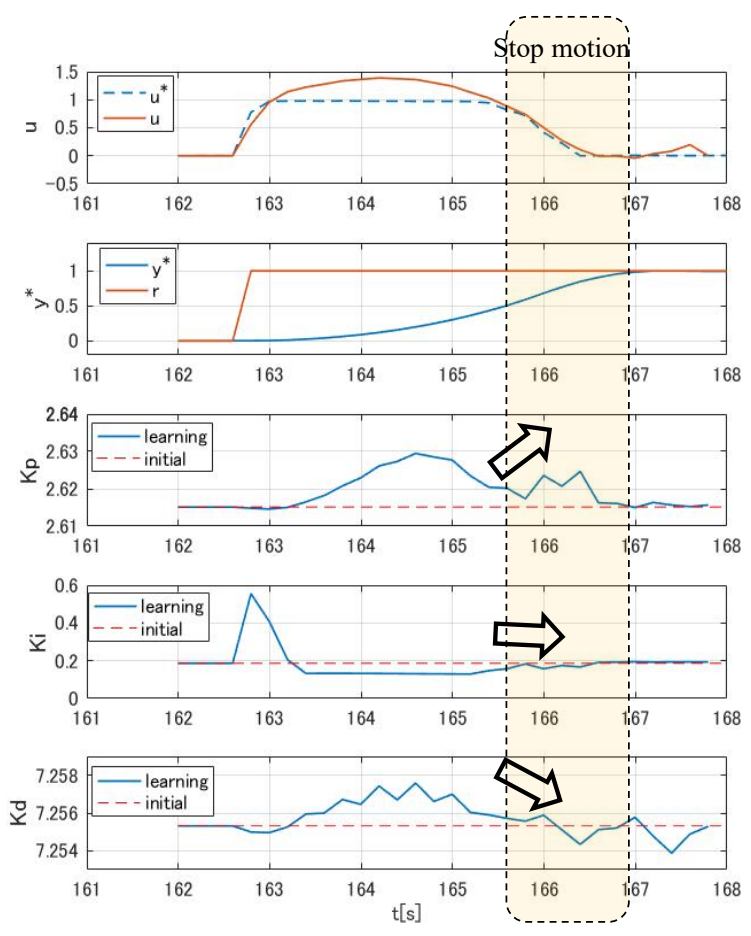

Fig. 5 Novice Skill Feature Extraction

verified based on PID gains. Especially, derivative gains of the professional and the novice are different. The change of derivative gain shows the future prediction to create the fluidity. Moreover, the fluidity of the operation is verified by the value of the input change. In the future work, the control row will be developed focused on operation fluidity.

\section{References}

1. T.Kakizaki, 'i-Construction (in Japanese)" monthly Journal Kensetsu, Vol.60, No.1, (2016), pp. 6-9

2. T.Yamamoto, S.Mori and A.Sakaguchi, Data-Driven Skill-Based PID Control of a Pilot-Scale Helicopter Model. , Int. J. of Innovative Computing Information and Control, Vol.4, No.12, (2008), pp.3349-3358

3. Y.Liao and T.Yamamoto, Design and Experimental Evaluation of a Human Skill-Based PID Controller, J. of Robotics, Networking and Artificial Life, Vol.2, No.3, (2015), pp.140-143

4. K.Koiwai, T.Yamamoto, T.Nanjo, Y.Yamazaki and Y.Fujimoto, Data-driven human skill evaluation for excavator operation, Proc. of IEEE Conf. on Advanced Intelligent Mechatronics, Banff (2016), pp.482-487.

5. K.Koiwai, Y.Liao, T.Yamamoto, T.Nanjo, Y.Yamazaki and Y.Fujimoto, Feature Extraction for Excavator Operation Skill Using CMAC, J. of Robotics and Mechatronics, Vol.28, No.5, (2016), pp.715-721. 